(rs145588689) is present in $0.2 \%$ of the world population and in $0.3 \sim 04 \%$ of non-Finnish Europeans. It seems likely that the variant underwent selection during the period of urbanization during the Middle Ages. The increased interferon responses may have enhanced survival against pandemic viruses. Whether P193A increases risk of systemic lupus erythematosus is unknown.

\section{A HUMAN SLE VARIANT NCF1-R90H PROMOTES KIDNEY DAMAGE AND MURINE LUPUS THROUGH ENHANCED TFH2 RESPONSES INDUCED BY DEFECTIVE EFFEROCYTOSIS OF MACROPHAGES}

\begin{abstract}
1,2 Lin-Yu Geng, ${ }^{1}$ Jian Zhao, ${ }^{1} Y u n$ Deng, ${ }^{1}$ Ivan Molano, ${ }^{1}$ Xue Xu, ${ }^{1}$ Ling-Xiao Xu, ${ }^{3}$ Phillip Ruiz, ${ }^{4}$ Quan-Zhen Li, ${ }^{2}$ Xue-Bing Feng, ${ }^{5}$ Miao-Jia Zhang, ${ }^{5}$ Wen-Feng Tan, 'Diane Kamen, ${ }^{6}$ Sang-Cheol Bae, ${ }^{1,7}$ Gary S Gilkeson, ${ }^{2}$ Ling-Yun Sun, 'Betty P Tsao*. ${ }^{1}$ Division of Rheumatology and Immunology, Medical University of South Carolina, SC, USA; ${ }^{2}$ Department of Rheumatology and Immunology, the Affiliated Drum Tower Hospital of Nanjing University Medical School, 321 Zhongshan Road, Nanjing 210008, China; ${ }^{3}$ Department of Surgery, University of Miami School of Medicine, FL, USA; ${ }^{4}$ Department of Immunology and Internal Medicine, University of Texas Southwestern Medical Center, Texas, USA; ${ }^{5}$ Department of Rheumatology, the First Affiliated Hospital of Naniing Medical University, 300 Guangzhou Road, Nanjing 210029, China; ${ }^{6}$ Department of Rheumatology, Hanyang University Institute for Rheumatology Research, Seoul, Republic of Korea
\end{abstract}

\subsection{6/lupus-2021-lupus21century.89}

Background We previously identified a p.Arg90His (p.R90H) hypomorphic variant of neutrophil cytosolic factor 1 (NCF1, a regulatory subunit of phagocyte $\mathrm{NADPH}$ oxidase 2 complex, NOX2) predisposes to multiple autoimmune diseases including systemic lupus erythematosus (SLE). We established a C57BL/6 (B6) mouse model with a knock-in (KI) H90 variant in the Ncf1 locus by CRISPR/Cas9 editing to study how this common NCF1 variant promotes the development of lupus manifestations.

Materials and Methods Wild type (WT) and KI littermates were assessed either for spontaneously-developed or pristaneinduced immune profiles and lupus-like features. Efferocytosis was assessed using irradiated WT thymocytes or Jurkat cells as apoptotic cells (AC) to co-culture with murine bone marrowderived macrophages or human circulating monocyte-derived macrophages, respectively. Disease activity and renal damage of SLE patients were assessed by SLEDAI and renal items of SLICC, respectively.

Results Compared to WT littermates, 5-week-old homozygous KI mice had reduced oxidative burst, splenomegaly, elevated type I interferon (IFN-I) scores, increased ratios of splenic follicular $\mathrm{T}$ helper 2 (Tfh2) to either $\mathrm{T}$ follicular regulatory ( $\mathrm{Tfr}$ ) or Tfh1 cell numbers, increased $\mathrm{ANA}^{+}$follicular, germinal center B cells and plasma cells, but no spontaneous kidney disease up to one-year of age. Pristane treatment induced kidney disease development in 36-week-old H90 KI B6 female mice, exhibiting increased Tfh2 coupled with decreased Tfr and Tfh1 proportions, robust germinal center formation and IgG autoantibody production. Decreased efferocytosis of macrophages derived from KI mice and homozygous H90 SLE patients promoted elevated ratios of Tfh2/Tfr and Tfh2/Tfh1 as well as dysregulated humoral responses due to reduced Hv1-dependent acidification of phagosome $\mathrm{pH}$ to neutralize the decreased electrogenic effect of the H90 variant, resulting in impaired maturation and proteolysis of phagosome. SLE patients carrying homozygous H90 genotype had elevated circulating $\mathrm{Tfh} 2 / \mathrm{Tfr}$ and $\mathrm{Tfh} 2 / \mathrm{Tfh} 1$ ratios, positive correlations of circulating Tfh2 percentage with plasmablast frequency and disease activity, deposition of $\operatorname{IgG}$ and complement C3 in kidney biopsies, and increased kidney damage in multiple ethnic populations.

Conclusion The same links between the NCF1 H90 hypofunctional genotype to lupus-like phenotype in a mouse model and SLE patients demonstrates it is the causal variant in the NCF1 locus associated with SLE.

Acknowledgements This study was supported by Lupus Research Alliance (grant to Betty Tsao) and NRF2017M3A9B40500335 (grant to Sang-Cheol Bae).

\section{THE RELATIONSHIP BETWEEN DNA METHYLATION PATTERNS AND DISEASE ACTIVITY IN A LONGITUDINAL MULTI-ANCESTRAL COHORT OF LUPUS PATIENTS}

\begin{abstract}
${ }^{1,2}$ Patrick Coit, ${ }^{1}$ Lourdes Ortiz-Fernandez, ${ }^{3}$ Emily E Lewis, ${ }^{3} \mathrm{~W}$ Joseph McCune, ${ }^{4}$ Kathleen Maksimowicz-McKinnon, ${ }^{1,5,6,7} \mathrm{Amr} H$ Sawalha*. ${ }^{1}$ Division of Rheumatology, Department of Pediatrics, University of Pittsburgh, Pittsburgh, PA, USA; ${ }^{2}$ Graduate Program in Immunology, University of Michigan, Ann Arbor, MI, USA; ${ }^{3}$ Division of Rheumatology, Department of Internal Medicine, University of Michigan, Ann Arbor, MI, USA; ${ }^{4}$ Division of Rheumatology, Henry Ford Health System, Detroit, MI, USA; ${ }^{5}$ Division of Rheumatology and Clinical Immunology, Department of Medicine, University of Pittsburgh, PA, USA; ${ }^{6}$ Lupus Center of Excellence, University of Pittsburgh School of Medicine, Pittsburgh, PA, USA; ${ }^{7}$ Department of Immunology, University of Pittsburgh, Pittsburgh, PA, USA
\end{abstract}

\subsection{6/lupus-2021-lupus21century.90}

Background Epigenetic dysregulation is implicated in the pathogenesis of lupus. We performed a longitudinal analysis of DNA methylation in lupus patients and assessed epigenetic changes over time and across disease activity status. Combining genetic and epigenetic analyses, we also examined ancestry-specific DNA methylation and DNA methylation changes influenced by genetic variants across the genome.

Methods A total of 54 female lupus patients, including 32 European-American and 22 African-American, were followed for up to 4 years. Blood samples were obtained at routine follow up visits and during disease flares, with a total of 229 samples collected. Disease activity at each blood draw was determined by SLEDAI. Granulocytes were isolated and DNA extracted. Genotyping was performed using the Infinium Global Screening Array v2.0, and genome-wide DNA methylation was assessed at each time-point using the Infinium MethylationEPIC array. Ancestry-specific DNA methylation changes and methylation quantitative trait loci (meQTL) were identified. A linear mixed effects model was implemented to identify DNA methylation alterations that vary with disease activity and the development of lupus nephritis during follow up.

Results We identified 487 hypomethylated and 420 hypermethylated $\mathrm{CpG}$ sites in African-American compared to European-American lupus patients, annotated to 391 and 316 unique genes, respectively. Differentially methylated genes include type I interferon-response genes such as IRF7 and IFI44, and genes related to the NFkB pathway. After adjusting for age, medications, and genetic background, DNA methylation levels in $142(15.7 \%)$ differentially methylated sites were found to be allele-specific and influenced by at least one genetic variant located within $1 \mathrm{~kb}$. TREML4, which plays a vital role in toll-like receptor signaling, was hypomethylated in African-American patients and demonstrated a strong cismeQTL association $\left(R^{2}=0.91\right)$. The associated genetic variant (rs9369265) significantly differs in allele frequencies between 
African-American and European-Americans, and is located within an active enhancer region in neutrophils and modifies TREML4 expression. In vitro patch methylation experiments confirmed the regulatory effects of TREML4 methylation upon gene expression. Experiments to assess the functional effects of TREML4 overexpression in human neutrophils are underway in our laboratory. Interestingly, the DNA methylome was highly stable across disease activity levels and over time. Two sites cg26104306 (SNX18; FDR-adjusted P-value $=3.38 \mathrm{x}$ $10^{-2}$ ) and $\operatorname{cg} 06708913$ (FDR-adjusted P-value $=3.43 \times 10^{-2}$ ) were associated with changing disease activity levels in African-American patients. Demethylation of a $\mathrm{CpG}$ site located within GALNT18 was associated with the development of active lupus nephritis.

Conclusion Lupus granulocytes demonstrate significant differences in DNA methylation patterns between African-American and European-American patients. DNA methylation profiles in lupus patients are influenced by ancestry-specific genetic variants and are highly stable over time independent of disease activity levels. Progressive demethylation in SNX18 was observed with increasing disease activity in granulocytes from African-American lupus patients, and demethylation in GALNT18 was associated with the development of lupus nephritis in our cohort during follow up.

\section{SINGLE-CELL EPIGENETIC PROFILING HIGHLIGHTS GENETIC IMPACT ON CHROMATIN ACCESSIBILITY IN SLE}

1,2,3 3 ai Ma, ${ }^{4}$ Richard C Pelikan, ${ }^{4}$ Yao Fu, ${ }^{4}$ Jennifer A Kelly, ${ }^{4}$ David Murphy, ${ }^{4}$ Graham B Wiley, ${ }^{3}$ Vinay K Kartha, ${ }^{5}$ Caleb Lareau, ${ }^{2,3}$ Jason D Buenrostro, ${ }^{4}$ Patrick M Gaffney*. ${ }^{1}$ Department of Biology, MIT, Cambridge, MA, USA; ${ }^{2}$ Broad Institute of MIT and Harvard, Cambridge, MA, USA; ${ }^{3}$ Department of Stem Cell and Regenerative Biology, Harvard University, Cambridge, MA, USA; ${ }^{4}$ Genes and Human Disease Research Program, Oklahoma Medical Research Foundation, Oklahoma City, OK, USA; ${ }^{5}$ Department of Pathology, Stanford University, Stanford, CA, USA

\subsection{6/lupus-2021-lupus21century.91}

Background Chromatin accessibility (CA) is a critical epigenetic feature identifying genomic loci that actively participate in gene-regulating functions, such as transcription and DNA repair. In turn, genetic polymorphisms within these loci can affect the magnitude of CA, i.e. a chromatin accessibility quantitative trait locus (caQTL). Changes in CA have been implicated in inflammatory disease, and previous genetic research has identified several risk haplotypes for systemic lupus erythematosus (SLE). However, it remains unclear how CA may interact with genetic risk factors in SLE pathogenesis. To better understand how CA in SLE may be driven by genetic variation, we performed single-cell assay for transposase accessible chromatin (sciATAC-seq) on peripheral blood mononuclear cells (PBMCs). SciATAC-seq is an efficient, scalable and sensitive assay that allows the epigenetic profiling of thousands of cells from an individual. By combining these profiles with genotypic data, we can search for caQTLs as evidence of genetic-epigenetic interaction specific to SLE.

Methods PBMCs were isolated from 45 SLE patients and 50 healthy controls. Each PBMC sample underwent both genotyping and sci-ATAC sequencing. DNA was then sequenced by Illumina Next-seq. Cell-specific ATAC reads were demultiplexed and quantified by custom software developed by the BROAD Institute (Cambridge, MA). Genotyping data was phased and imputed using the IMPUTE2 tool suite. Cell typespecific caQTL analysis was performed by RASQUAL.

Results An average of 980 cells were sequenced per sample, with a total of 745,697 CA sites measured. We identified a total of 153,716 caQTL relationships across 17 distinct immune cell types, involving 59,715 unique variants; 59\% of which are also reported as expression QTLs in whole blood. The majority of caQTLs already implicated in autoimmune disease risk haplotypes occurred predominantly in B cells and plasmacytoid dendritic cells. CA profiles exhibit cell type-specific cluster orientation highly correlated with caQTL genotype. Genotypes at variant rs1131665, previously associated with an SLE risk haplotype in IRF7 and here as a caQTL, distinguished subpopulations of $\mathrm{B}$ cells and monocytes on the basis of global CA profiles. Increases of CA at the variant were present in SLE individuals compared to controls, suggesting caQTL variants contribute to a genome-wide epigenetic phenotype for SLE risk.

Conclusion Using advances in single-cell epigenetic profiling, we were able to identify thousands of genetic variants which influence epigenetic functions, in a cell type-specific way, through their association to CA. Understanding the molecular mechanisms for how caQTLs alter cell type-specific chromatin accessibility will provide new insights into the role of epigenetic regulation in SLE pathogenesis.

Acknowledgments The research reported in this abstract was supported by Institutional Development Awards (IDeA) from the National Institute of General Medical Sciences (U54GM104938 and P30GM110766), the National Institute of Arthritis and Musculoskeletal and Skin Diseases (P30AR073606 and R01AR073750); the National Institute of Allergy and Infectious Diseases (UM1AI144292 and R01AI156724), and by the Presbyterian Health Foundation (OKC, OK).

\section{DIFFERENCES IN CHROMATIN ARCHITECTURE PRE- AND POST-INDUCTION THERAPY IN PEDIATRIC LUPUS PATIENTS}

1,2,3 Joyce S Hui-Yuen ${ }^{*},{ }^{4,5}$ Kaiyu Jiang, ${ }^{3}$ Susan Malkiel, ${ }^{3}$ Betty Diamond, ${ }^{4,5}$ James N Jarvis. 'Division of Pediatric Rheumatology, Steven and Alexandra Cohen Children's Medical Center, Lake Success, NY, USA; ${ }^{2}$ Department of Pediatrics, Hofstra-Northwell School of Medicine, Hempstead, NY, USA; ${ }^{3}$ Center for Autoimmune, Musculoskeletal, and Hematologic Diseases Research, Feinstein Institute for Medical Research, Manhasset, NY, USA; ${ }^{4}$ Department of Pediatrics, University at Buffalo, Buffalo, NY, USA; ${ }^{5}$ Genetics, Genomics, and Bioinformatics Program, University at Buffalo, Buffalo, NY, USA

10.1136/lupus-2021-lupus21century.92

Background Systemic lupus erythematosus (SLE) may be triggered by gene-environment interactions. Data remain scarce on how epigenetic variance contributes to disease risk in pediatric SLE (pSLE). Our objectives were to identify differences in chromatin architecture in treatment-naïve pSLE compared to healthy children (HC) and pSLE patients after induction therapy.

Methods We used assays for transposase-accessible chromatinsequencing (ATACseq) in 8 pSLE patients pre- and post-induction therapy and $5 \mathrm{HC}$ to investigate whether regions of open chromatin unique to pSLE patients demonstrate enrichment for transcriptional regulators, using standard computational approaches and a false discovery rate of $<0.05$.

Results The mean age of onset was 13.75 (range 7-17) years in pSLE, and mean SLEDAI was 12.8 (range 6-24). We 